\title{
Sensory innervation around immediately vs. delayed loaded implants: a pilot study
}

\author{
Yan Huang ${ }^{1,2}$, Jeroen van Dessel ${ }^{1}$, Wendy Martens ${ }^{3}$, Ivo Lambrichts ${ }^{3}$, Wei-Jian Zhong ${ }^{4}$, Guo-Wu Ma ${ }^{4}, \operatorname{Dan~Lin~}^{5}$, \\ Xin Liang ${ }^{4}$ and Reinhilde Jacobs ${ }^{1}$
}

\begin{abstract}
Although neurophysiological and psychophysical proof of osseoperception is accumulating, histomorphometric evidence for the neural mechanisms of functional compensation following immediate and delayed implant loading is still lacking. For this randomized split-mouth study, six mongrel dogs randomly received one of four treatment protocols at 36 implant-recipient sites over 16 weeks (third maxillary incisor, third and fourth mandibular premolar): immediate implant placement and immediate loading (IIP+IL); delayed implant placement and delayed loading (DIP+DL); delayed implant placement and immediate loading (DIP+IL); and natural extraction socket healing (control). Histomorphometry was performed in the peri-implant bone and soft tissues within $\mathbf{3 0 0} \mu \mathrm{m}$ around the implants. Immunocytochemistry and transmission electron microscopy were used to confirm the presence of neural structures and to reveal their ultrastructural characteristics, respectively. Myelinated nerve fibres densely populated the peri-implant crestal gingival and apical regions, although they were also identified in the woven bone and in the osteons near the implant threads. Compared with the control group in the mandible, the group that received IIP $+\mathrm{IL}$ showed a higher innervation (in $\mathrm{N} \cdot \mathrm{mm}^{-2}, 5.94 \pm 1.12 \mathrm{vs} .3 .15 \pm 0.63$, $P<0.001$ ) and smaller fibre diameter (in $\mu \mathrm{m}, 1.37 \pm 0.05$ vs. $1.64 \pm 0.13, P=0.016$ ), smaller axon diameter (in $\mu \mathrm{m}, 0.89 \pm 0.05 v s$. $1.24 \pm 0.10, P=0.009)$ and g-ratio $(0.64 \pm 0.04$ vs. $0.76 \pm 0.05, P<0.001)$ in the middle region around the implants. Compared with DIP+IL in the mandible, IIP+IL had a higher nerve density (in $\mathrm{N} \cdot \mathrm{mm}^{-2}, 13.23 \pm 2.54$ vs. $9.64 \pm 1.86, P=0.027$ ), greater fibre diameter (in $\mu \mathrm{m}, 1.32 \pm 0.02$ vs. $1.20 \pm 0.04, P=0.021$ ), greater axon diameter (in $\mu \mathrm{m}, 0.92 \pm 0.01$ vs. $0.89 \pm 0.03, P=0.035$ ) and lower g-ratio $(0.69 \pm 0.01$ vs. $0.74 \pm 0.01, P=0.033)$ in the apical region around the implants. It may be assumed that the treatment protocol with IIP+IL is the preferred method to allow optimized peri-implant re-innervation, but further functional measurements are still required.

International Journal of Oral Science (2014) 7, 49-55; doi:10.1038/ijos.2014.53; published 12 September 2014
\end{abstract}

Keywords: animal experiments; dental implants; histomorphometry; immediate loading; mechanoreceptor; myelinated nerve fibres; osseoperception

\section{INTRODUCTION}

Periodontal mechanoreceptors play a crucial role during oral function. ${ }^{1-2}$ After tooth extraction, the type of amputation, oral motor behaviour and natural biting function ${ }^{3}$ may be changed as a consequence of excessive damage to periodontal mechanoreceptors and intradental nociceptors. ${ }^{4}$

Inspired by the report of lower limb amputees with bone-anchored prostheses ${ }^{5}$ who are able to differentiate between walking on different soils, the concept of 'osseoperception' emerged. ${ }^{6-8}$ Psychophysical and neurophysiological studies indicated (partial) recovery of tactile function $^{9-11}$ and even activation of the primary sensorimotor cortex upon dental implant stimulation, ${ }^{12-13}$ suggesting a restoration of the sensory feedback pathway in humans.

Meanwhile, histological evidence revealed the presence of specialized Ruffini mechanoreceptive terminals in the immediate vicinity of the implants ${ }^{14}$ and in the peri-implant epithelium, ${ }^{15-16}$ which were predominantly derived from myelinated fibres. Moreover, researchers identified abundant unmyelinated nerve fibres under the implant thread area, with implant loading increasing the number of free nerve endings. ${ }^{17}$ Although myelinated nerve fibres are known to be involved in numerous neurophysiological functions, the quantitative observation of these periimplant fibres is rarely addressed or characterized histomorphologically. Furthermore, there are almost no data available on the influence of extraction and implant loading on nerve regeneration and activation.

Therefore, the aim of this study is to histomorphometrically assess the effects of immediate and delayed implant loading on peri-implant innervation in a randomized split-mouth trial.

\section{MATERIALS AND METHODS}

Study design

The protocol of this animal experiment was approved by the bioethics committees of the Dalian Medical University, the Hasselt University

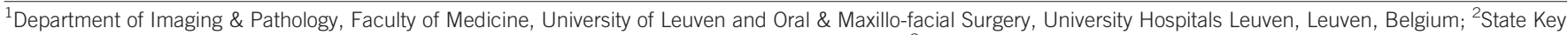

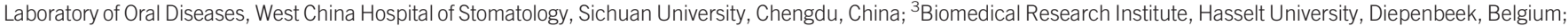
${ }^{4}$ School of Stomatology, Dalian Medical University, Dalian, China and ${ }^{5}$ Center for Statistics, Hasselt University, Diepenbeek, Belgium 
and KU Leuven (P059-2012-TK) and complied with the ARRIVE guidelines for preclinical studies (Supplementary Information). To reduce the number of animals used in the experiment and to avoid individual differences or even inter-subject regional heterogeneity, a split-mouth randomized design using four treatment protocols in six tooth positions of six male mongrel dogs was applied (Table 1). The investigators ensured the maximum degree of unpredictability of the allocation sequence by coin toss prior to the surgeries. The sample size calculation was based on our previous study on dogs. ${ }^{18}$ Briefly, six healthy male mongrel dogs (weight: $14.8-18.1 \mathrm{~kg}$, age: $20-24$ months) without any oral health or systemic diseases were included and housed individually in indoor cages. The dogs' diet during the course of the experiment (including whole grain flour, corn meal, soybean cake, fishbone meal and eggs) abided by the general feeding program at the Experimental Animal Center of Dalian Medical University, China.

\section{Surgical procedure}

Before the surgery, all animals received 1 week of antibiotic prophylaxis (gentamicin sulphate, $1600000 \mathrm{U} \cdot$ day $^{-1}$; Lingrui Pharmaceutical, Zhengzhou, China). Custom-made threaded titanium implants (Grade $\mathrm{V}$, machined surface, $3.1 \mathrm{~mm}$ diameter for the third maxillary incisors and $4.1 \mathrm{~mm}$ diameter for the third and fourth mandibular premolars, $L=11 \mathrm{~mm}$ long) were manufactured and sterilized for surgery. The surgical procedures were performed under general anaesthesia with Sumianxin $\left(0.1 \mathrm{~mL} \cdot \mathrm{kg}^{-1}\right.$ xylazine hydrochloride; Changchun Military Academy of Medical Sciences, Changchun, China), while local anaesthesia (2-4 mL lidocaine 2\% with epinephrine 1:100000; Tianjin Pharmaceutical, Tianjin, China) was used only at the surgical sites. Postoperatively, the animals were given antibiotic treatment (gentamycin sulphate $300 \mathrm{mg}$; Tianjin Pharmaceutical, Tianjin, China) and analgesics (ibuprofen $400 \mathrm{mg}$; Tianjin Pharmaceutical, Tianjin, China) intramuscularly for 3 days. Each implant recipient site was randomly assigned to one of four treatment protocols that are frequently used clinically (Figure 1), and a general two-stage implant placement was applied accordingly: (i) immediate implant placement and immediate loading (IIP+IL); (ii) delayed implant placement (8 weeks after tooth extraction) and delayed loading (4 weeks after implant placement) (DIP $+\mathrm{DL})$; (iii) delayed implant placement (8 weeks after tooth extraction) and immediate loading (DIP+IL); and (iv) control group, normal extraction socket healing. The intervals between the implant placement surgery and the animal euthanasia were all 8 weeks for the IIP+IL, DIP+DL and DIP+IL groups. One surgeon (WZ) with a decade of clinical experience in implant dentistry performed the entire surgical procedure on each subject. He was kept blinded to the allocation process until tooth extraction had been

Table 1 Random distribution of split-mouth design in six experimental dogs

\begin{tabular}{|c|c|c|c|c|c|c|}
\hline Teeth & 1\# dog & 2\# dog & 3\# dog & 4\# dog & 5\# dog & $6 \#$ dog \\
\hline I (R) & Control & $\mathrm{DIP}+\mathrm{DL}$ & $\mathrm{DIP}+\mathrm{IL}$ & $I I P+I L$ & Control & $D I P+D L$ \\
\hline I (L) & $\mathrm{DIP}+\mathrm{DL}$ & $\mathrm{DIP}+\mathrm{IL}$ & $I I P+I L$ & Control & $\mathrm{DIP}+\mathrm{DL}$ & $\mathrm{DIP}+\mathrm{IL}$ \\
\hline P3 (R) & $\mathrm{DIP}+\mathrm{DL}$ & $D I P+I L$ & $I I P+I L$ & Control & $\mathrm{DIP}+\mathrm{DL}$ & $D I P+I L$ \\
\hline P3 (L) & $D I P+I L$ & $I I P+I L$ & Control & $\mathrm{DIP}+\mathrm{DL}$ & $D I P+I L$ & $I I P+I L$ \\
\hline P4 (R) & Control & $\mathrm{DIP}+\mathrm{DL}$ & DIP+IL & $I I P+I L$ & Control & $\mathrm{DIP}+\mathrm{DL}$ \\
\hline P4 (L) & $\| \mathrm{IP}+\mathrm{IL}$ & Control & $D I P+D L$ & DIP+IL & $\| P+I L$ & Control \\
\hline
\end{tabular}

DIP, delayed implant placement; DL, delayed loading; I, third maxillary incisors; IIP, immediate implant placement; IL, immediate loading; L, left side; P3, third mandibular premolars; $\mathrm{P} 4$, fourth mandibular premolars; $\mathrm{R}$, right side. In total, 36 samples were used in the study (control=9; IIP $+I L=8 ; D I P+D L=10$; $\mathrm{DIP}+\mathrm{IL}=9$ ). completed, as he could no longer be blinded to the further allocated implant placement.

\section{Occlusion restoration}

The shoulder of the implant was placed at the level of the marginal bone under the same surgical conditions as the tooth extractions in terms of sterility, operation room and anaesthesia. Primary stability of the implants was confirmed by percussion testing. Customized posts with a Ni-Ti crown (College of Stomatology, Dalian Medical University, Dalian, China) were then set using resin cement (RelyX, Unicem, RX; 3M ESPE, St Paul, MN, USA). After the post-crown restoration, the edge-to-edge occlusion contacts during chewing between the implant-recipient sites and antagonist teeth were extensively assured. The loading pressure on the implants was kept similar using $0.02-\mathrm{mm}$ articulating papers during the experimental period (Accufilm II, RX; 3M ESPE, St Paul, MN, USA). Plaque control was ensured at least once per week using a $0.2 \%$ chlorhexidine gel with a soft toothbrush.

\section{Animal euthanasia and histology}

At the sixteen week, euthanasia was performed with an overdose of xylazine hydrochloride (intravenous injection), and all animals were immediately perfused with $4 \%$ paraformaldehyde and $0.0125 \%$ glutaraldehyde in $0.1 \mathrm{~mol} \cdot \mathrm{L}^{-1}$ phosphate buffer ( $\mathrm{pH}$ 7.4). Specimen blocks with a 3- to $5-\mathrm{mm}$ piece of peri-implant bone and gingiva tissue were retrieved and immersed in $0.5 \mathrm{~mol} \cdot \mathrm{L}^{-1}$ ethylenediaminetetraacetic acid (EDTA) phosphate-buffered saline $(\mathrm{pH} 7.4)$ at $4{ }^{\circ} \mathrm{C}$ for 10 months, allowing the implants to be easily removed from the specimens using surgical forceps. Following dehydration and embedding in paraffin, thin serial sections $(\sim 6 \mu \mathrm{m})$ were prepared along the mesialdistal direction in the middle of the implant or extraction area, considering the vertical loading at the implant-recipient sites and abundant trabecular bone structures in the mesial-distal direction (more trabecular bone means more space for the development of neuroreceptors). All sections were stained with Masson's trichrome stain.

\section{Immunohistochemistry}

Immunohistochemistry was performed on the same serial sections in each group to confirm the presence of myelinated nerve structures using the labelled avidin-biotin method, as previously described. ${ }^{19}$ After fixation, embedded sections were deparaffinized. Subsequently, the sections were microwaved in $10 \mathrm{mmol} \cdot \mathrm{L}^{-1}$ citrate buffer, $\mathrm{pH} 6.0$, and endogenous peroxidase activity was quenched with $0.5 \% \mathrm{H}_{2} \mathrm{O}_{2}$. Nonspecific binding sites were blocked with $10 \%$ normal goat serum. Then, the sections were stained with primary antibody mouse monoclonal anti-neurofilament (NF, 1:1000; Abcam, Cambridge, UK) or goat monoclonal anti-neuropeptide Y (NPY, 1:1000; Santa Cruz Biotechnology, CA, USA) followed by incubation with peroxidase-conjugated anti-mouse/goat IgG (Dako Cytomation, Glostrup, Denmark) for $30 \mathrm{~min}$. Sections were counterstained with Mayer's haematoxylin, coverslipped with an aqueous mounting medium and examined using a photomicroscope equipped with an automated camera (Nikon Eclipse 80i; Nikon, Tokyo, Japan). Controls from adjacent sections were subjected to the same staining with omission of the primary antibody.

\section{Transmission electron microscopy}

To analyse the ultrastructural characteristics of the nerve fibres, one decalcified sample from each group was randomly selected after isolation of the implants. Following fixation with $2 \%$ glutaraldehyde in $0.05 \mathrm{~mol} \cdot \mathrm{L}^{-1}$ cacodylate buffer ( $\mathrm{pH} 7.3$ ), samples were post-fixed in 


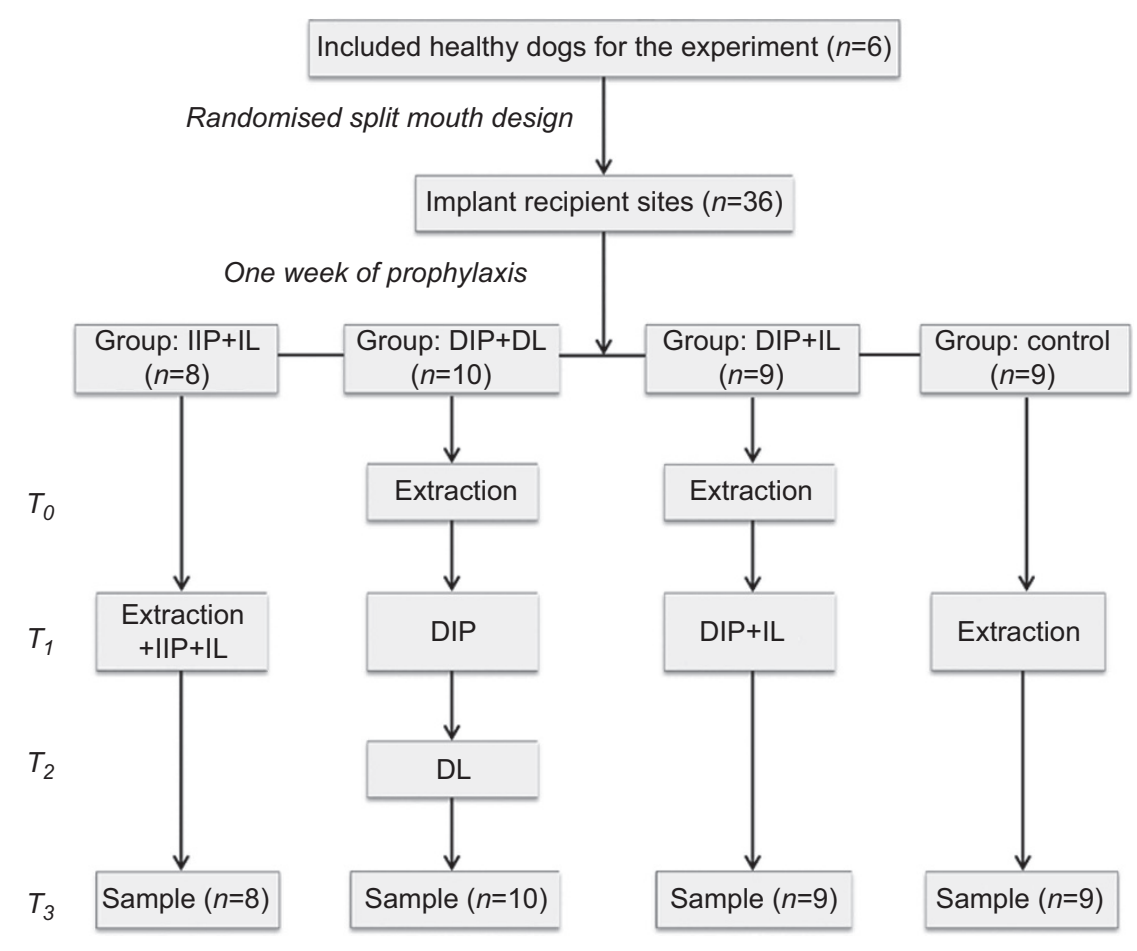

Figure 1 Flow chart of study design and timetable. IIP +IL (immediate implant placement and immediate loading); DIP +DL (delayed implant placement and delayed loading); DIP+IL (delayed implant placement and immediate loading); control (normal extraction socket healing); T0, baseline; T1, 8 weeks; T2, 12 weeks; T3, 16 weeks.

$2 \%$ osmium tetroxide for $1 \mathrm{~h}$ and stained with $2 \%$ uranyl acetate in $10 \%$ acetone for $20 \mathrm{~min}$. Subsequently, the samples were subjected to a dehydrating series of graded concentrations of acetone and embedded in araldite. Serial ultrathin sections $(\sim 0.06 \mu \mathrm{m})$ were mounted on $0.7 \%$ Formvar-coated copper grids, contrasted with $0.5 \%$ uranyl acetate and a stabilized solution of lead citrate, and examined in a transmission electron microscope (EM 208; Philips, Eindhoven, The Netherlands) operated at $80 \mathrm{kV}$. The microscope was provided with a Morada Soft Imaging System (Olympus, Tokyo, Japan) camera to acquire high-resolution images of the evaluated samples. The images were processed digitally with iTEM-FEI software (Morada Soft Imaging System; Olympus, Tokyo, Japan).

\section{Histomorphometric analysis}

Three serial sections from each sample were digitized using MiraxScan (Carl Zeiss, Göttingen, Germany) and were evaluated to obtain an overall mean value of the estimated parameter. One experienced observer (YH), who was blinded to implant groups, evaluated the density of nerve fibres $\left(\mathrm{N} \cdot \mathrm{mm}^{-2}\right.$, number of myelinated nerve fibres per area). A magnification of $\times 100$ with a 17 -inch LCD monitor (Dell, CA, USA ) and an image software package (Panoramic Viewer, Hungary) was used. Four regions of interest (ROIs) at a distance of $300 \mu \mathrm{m}$ from the implant were selected (Figure 2) as an approximate width of the periodontal ligament (PDL) because this is the region that is most affected by loading transmission through implants. ${ }^{20}$ The following morphological predictors of regeneration were further estimated in four ROIs (Figure 3) in the peri-implant bone and crestal gingiva tissues above placed implants: fibre diameter $(D, \mu \mathrm{m}$, the outer diameter of the myelinated fibre), axon diameter $(d, \mu \mathrm{m}$, the diameter of the axon proper) and g-ratio $(d / D$, a functional and structural index of optimal axonal myelination). ${ }^{21}$ To acquire maximal precision, the
$D$ and $d$ were measured using the 'lesser fibre diameter' method, defined as the maximum diameter across the lesser aspect of the nerve fibre and axon, to prevent any potential distortion. ${ }^{19}$ Partial fibres at the borderline of an ROI were excluded.

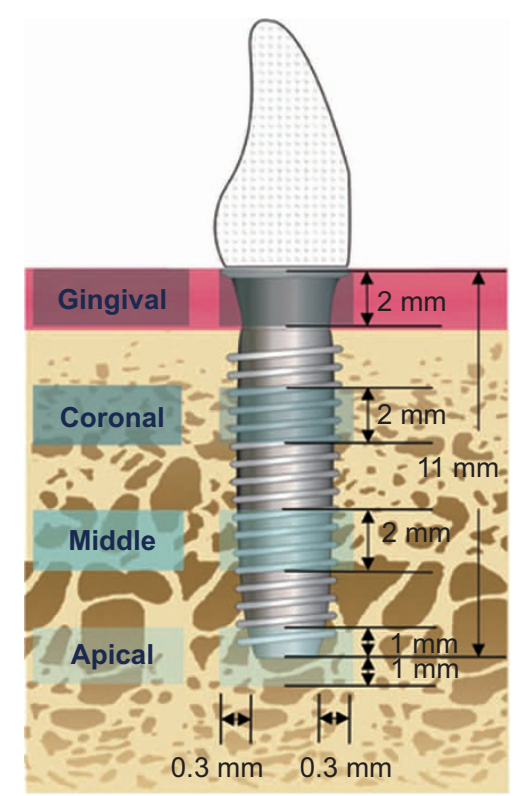

Figure 2 Schematic diagram of the ROIs for histomorphometrical analysis of peri-implant innervation. Four different ROls were displayed in gingival, coronal, middle and apical regions along the axis of the implant at a mesial-distal view ( width $=0.3 \mathrm{~mm}$; height $=2 \mathrm{~mm}$ for each region). ROI, region of interest. 


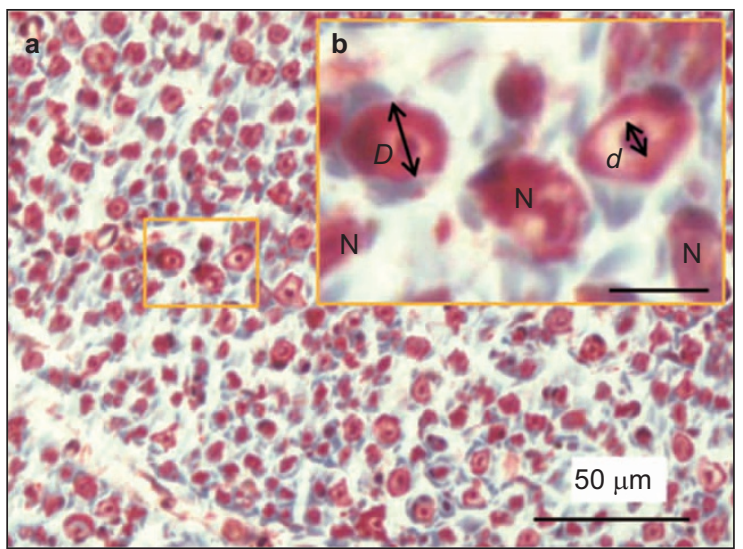

Figure 3 Morphometric analyses of the histological sections stained with Masson's trichrome stain (light microscopy). (a) Myelinated nerve fibres characterized by axons being surrounded by myelin sheaths formed by the Schwann cells; (b) a magnification of the selected box region in (a) showing the basic morphometric parameters of myelinated nerve fibres (scale bar $=10 \mu \mathrm{m}$ ). $d$, axon diameter; $D$, nerve fibre diameter; $\mathrm{N}$, nerve fibres.

\section{Statistical analysis}

To fully consider the potential fixed and random effects in the splitmouth design, the linear mixed model was used after appropriate transformation of the raw data, including the fixed effects of implant groups, jaws (upper vs. lower), regions (from gingiva to apex), their two-way and three-way interaction terms and the random effects of dogs and error terms with the number of measurements in each region and each dog as weights. The region and implant group comparisons were performed due to the significance of the three-way interaction effect (group $\times$ jaw $\times$ region). All hypothesis testing was implemented in SAS 9.2 at a significance level of 0.05 .

\section{RESULTS}

All animals recovered well and without any clinical signs of inflammation during the experimental period, and all implants were clinically stable until euthanasia. The peri-implant bone and soft tissue were healthy overall.

Histological observation revealed myelinated nerve fibres in the peri-implant crestal gingiva, the woven bone around the implants and the osteons near the implant threads (Figure 4a-4d). Nerve fibres were mainly distributed perivascularly with frequent branching and orientated along the axis of the blood vessels. These myelinated fibres were also located within natural extraction healing sockets and in the Haversian canal of an osteon (Figure 4e-4f). NF and NPY immunoreactivity (Figure $4 \mathrm{~g}-4 \mathrm{~h}$ ) further confirmed the existence of myelinated nerve fibres in the peri-implant bone and gingiva. Ultrastructurally (Figure 4i-4j), myelinated nerve fibres were occasionally accompanied by unmyelinated nerve fibres, and the peripherally oriented mitochondria were identified.

Gingival regions demonstrated a significantly greater $(P<0.04)$ amount of innervation than any peri-implant bone region (Figure 5), though this difference was not always significant in the maxilla. In the peri-implant bone regions, the nerve density increased in the apical direction with statistical significance $(P<0.02)$, except in the maxilla for the DIP+IL group $(P>0.05)$. There was also a trend towards higher nerve density around mandible implants than around maxilla implants, especially in the peri-implant gingiva of the three implant groups. Nerve density in the implant groups was higher compared with the control bone sites, especially in the mandible $(P<0.001)$. The
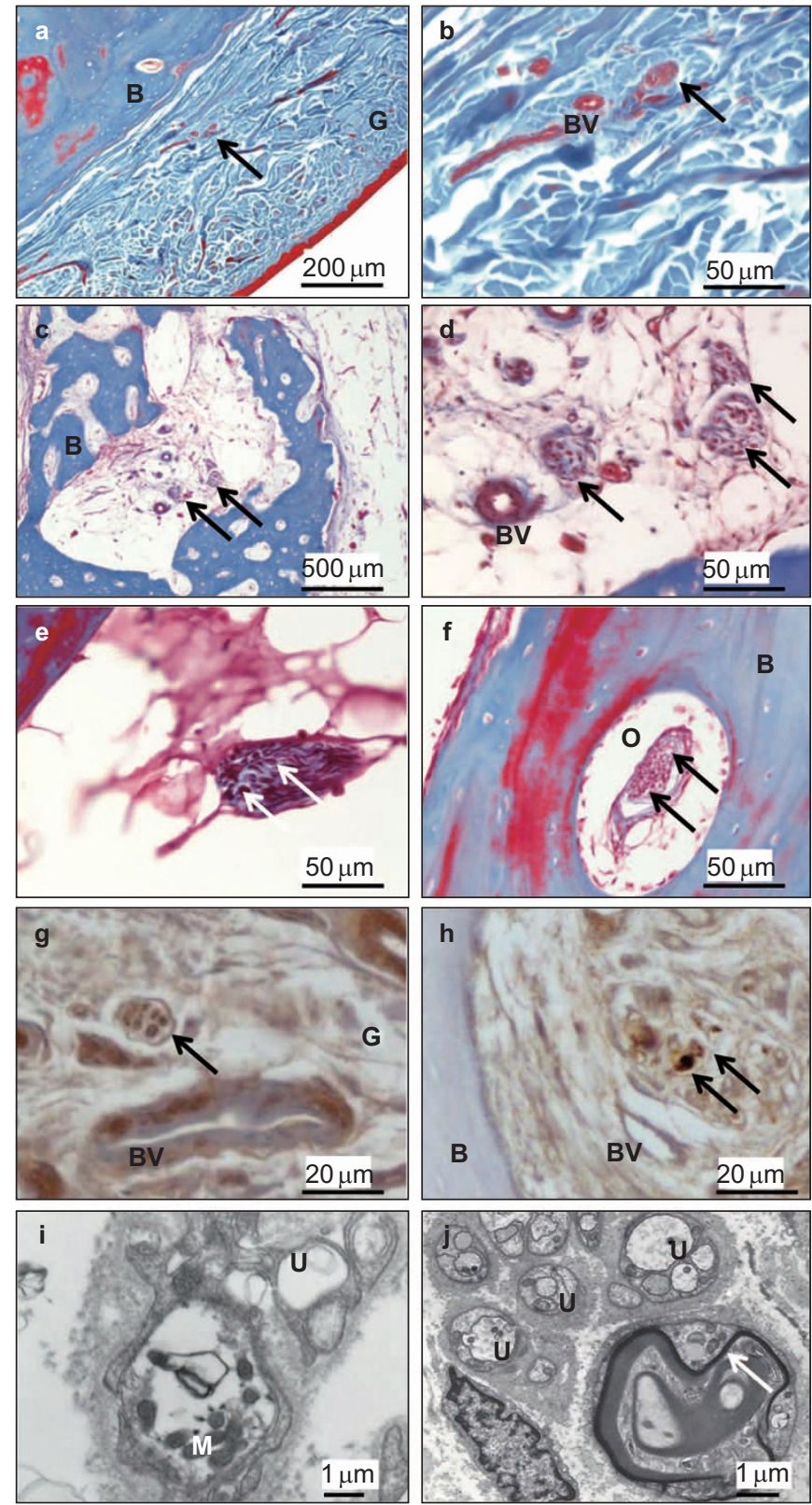

Figure 4 Decalcified sections stained with Masson's trichrome stain, neurofilament protein and neuropeptide $Y$. (a) Myelinated nerve fibres existed in the peri-implant crestal gingiva (DIP + IL group). (b) A magnification of (a) showing a bundle of nerve fibres and a blood vessel. (c) Myelinated nerve fibres presented in the woven bone area at the apical region (IIP +IL group). (d) A magnification of (c) showing several bundles of myelinated nerve fibres accompanied by a blood vessel. (e) A bundle of myelinated nerve fibres was observed at the middle region of a natural extraction healing socket (control group). (f) Myelinated nerve fibres were located in the Haversian canal of an osteon at the apical region (DIP +DL group). (g) Myelinated nerve fibres were identified in the crestal gingiva around the implants (control group); (h) crosssectioned myelinated nerve fibres were located in peri-implant bone tissue (control group). (i) Unmyelinated afferent nerve ending enveloped by a thin lamina of Schwann cell sheets and characterized by the peripheral localisation of mitochondria (IIP+IL group). (j) A bundle of unmyelinated and one myelinated nerve fibre in the peri-implant bone tissue (DIP+IL group). Arrows indicate myelinated nerve fibres; $\mathrm{B}$, bone; $\mathrm{BV}$, blood vessel; $\mathrm{G}$, gingiva; $\mathrm{M}$, mitochondria; O, osteon; $U$, unmyelinated nerve fibres. 
a

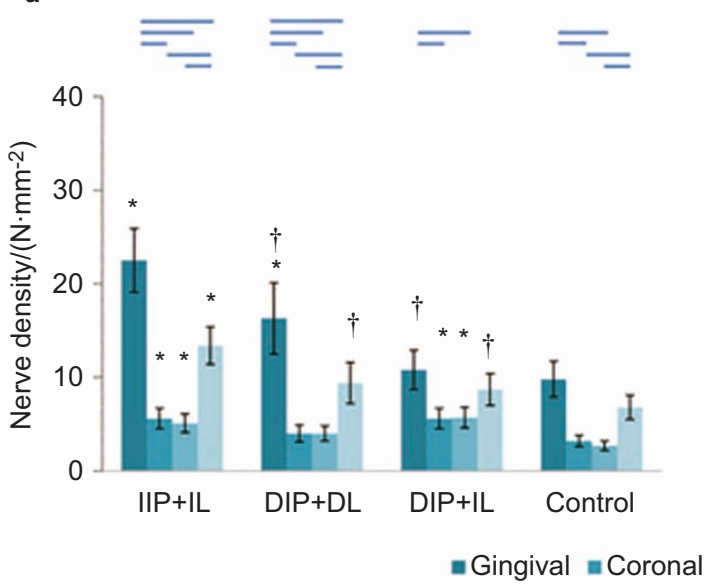

b

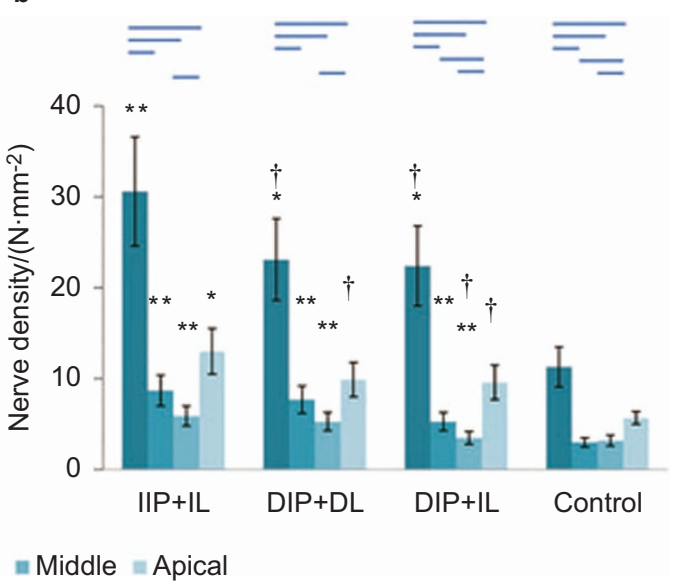

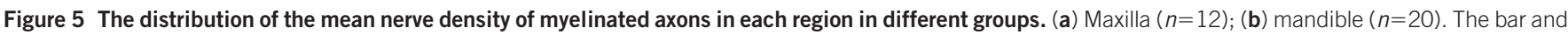

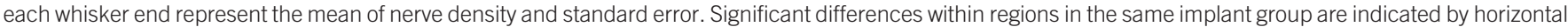

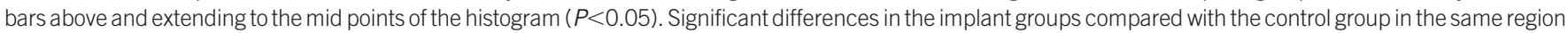
$(* P<0.05 ; * * P<0.001)$. Significant differences in other groups compared with the IIP + IL group in the same region ( $\left.{ }^{\dagger} P<0.05\right)$.

Table 2 Comparison of histomorphometric parameters of regenerated myelinated nerve fibres in peri-implant tissue for different groups

\begin{tabular}{|c|c|c|c|c|c|c|c|c|}
\hline \multirow[b]{2}{*}{ Histomorphometric perimeters } & \multicolumn{4}{|c|}{ Maxilla $(n=12)$} & \multicolumn{4}{|c|}{ Mandible $(n=20)$} \\
\hline & Gingiva & Coronal & Middle & Apical & Gingiva & Coronal & Middle & Apical \\
\hline \multicolumn{9}{|l|}{ Fibre diameter/ $\mu \mathrm{m}$} \\
\hline $\mathrm{IIP}+\mathrm{IL}$ & $1.23(0.02)$ & $1.42(0.08) *$ & $1.18(0.08)$ & $1.35(0.03)$ & $1.10(0.02) *$ & $1.38(0.05) *$ & $1.37(0.05)^{*}$ & $1.32(0.02)$ \\
\hline $\mathrm{DIP}+\mathrm{DL}$ & $1.20(0.05) * \dagger$ & $1.40(0.20) *$ & $1.07(0.18)^{* \dagger}$ & $1.34(0.05)$ & $1.09(0.02) *$ & $1.34(0.06)^{* \dagger}$ & $1.36(0.09) *$ & $1.29(0.02)$ \\
\hline $\mathrm{DIP}+\mathrm{IL}$ & $1.21(0.03)^{*}$ & $1.29(0.09)^{* \dagger}$ & $1.14(0.09)^{*}$ & $1.23(0.04)^{* \dagger \dagger}$ & $1.04(0.03) *$ & $1.14(0.07)^{* \dagger}$ & $1.22(0.13)^{* \dagger}$ & $1.20(0.04) * \dagger$ \\
\hline Control & $1.29(0.03)$ & $1.48(0.18)$ & $1.23(0.19)$ & $1.37(0.07)$ & $1.13(0.02)$ & $1.66(0.20)$ & $1.64(0.13)$ & $1.34(0.05)$ \\
\hline \multicolumn{9}{|l|}{ Axon diameter/ $\mu \mathrm{m}$} \\
\hline $\mathrm{IIP}+\mathrm{IL}$ & $0.87(0.02) *$ & $1.05(0.04) *$ & $0.87(0.05)$ & $0.98(0.02) *$ & $0.83(0.02) *$ & $0.94(0.03) *$ & $0.89(0.05) *$ & $0.92(0.01) *$ \\
\hline $\mathrm{DIP}+\mathrm{DL}$ & $0.86(0.03) *$ & $1.01(0.11) *$ & $0.80(0.05) *^{\dagger}$ & $0.97(0.03) *$ & $0.82(0.02) *$ & $0.93(0.05) *$ & $0.88(0.06)^{*}$ & $0.91(0.02) *$ \\
\hline $\mathrm{DIP}+\mathrm{IL}$ & $0.86(0.03) *$ & $0.92(0.05)^{* \dagger}$ & $0.86(0.05) *$ & $0.91(0.02)^{* \dagger \dagger}$ & $0.79(0.02)^{* \dagger}$ & $0.79(0.06)^{* \dagger}$ & $0.83(0.09)^{* \dagger}$ & $0.89(0.03) * \dagger$ \\
\hline Control & $0.97(0.02)$ & $1.18(0.12)$ & $0.93(0.13)$ & $1.08(0.05)$ & $0.88(0.02)$ & $1.17(0.15)$ & $1.24(0.10)$ & $1.03(0.03)$ \\
\hline \multicolumn{9}{|l|}{ g-ratio } \\
\hline$I I P+I L$ & $0.68(0.01)^{*}$ & $0.70(0.03) *$ & $0.74(0.03)$ & $0.72(0.01)^{*}$ & $0.75(0.01) *$ & $0.68(0.02) *$ & $0.64(0.04) * *$ & $0.69(0.01) * *$ \\
\hline $\mathrm{DIP}+\mathrm{DL}$ & $0.73(0.02)^{\dagger}$ & $0.75(0.08)^{\dagger}$ & $0.75(0.07)$ & $0.73(0.02) *$ & $0.75(0.01)^{*}$ & $0.69(0.03)$ & $0.67(0.03) * \dagger$ & $0.72(0.01)^{* * \dagger}$ \\
\hline $\mathrm{DIP}+\mathrm{IL}$ & $0.71(0.01)^{* \dagger}$ & $0.71(0.04) *$ & $0.75(0.04)$ & $0.74(0.01)$ & $0.76(0.01)$ & $0.69(0.03)$ & $0.68(0.05)^{* \dagger}$ & $0.74(0.01)^{* \dagger}$ \\
\hline Control & $0.75(0.01)$ & $0.80(0.07)$ & $0.76(0.07)$ & $0.79(0.03)$ & $0.78(0.01)$ & $0.71(0.08)$ & $0.76(0.05)$ & $0.77(0.02)$ \\
\hline
\end{tabular}

Values are shown as estimated means (s.e.). Significant differences ( $* P<0.05 ; * * P<0.001)$ between the implant groups and the control group in the same region. Significant differences $\left({ }^{\dagger} P<0.05 ;{ }^{\dagger} P<0.001\right)$ between other groups and the IIP+IL group in the same region.

IIP+IL group had the highest density in both jaws, though the significant differences of nerve density between three implant groups were mostly noted in the gingival and apical regions $(P<0.04)$. No difference was observed in the nerve density between the DIP $+D L$ and DIP + IL groups $(P>0.05)$.

Further morphological analyses on predictors of regeneration of myelinated nerve fibres indicated that all implant groups had slightly smaller fibre diameter when compared with the natural extraction and healing group (Table 2$)$, especially in the mandible $(P<0.02)$. The fibre and axon diameters in the IIP+IL group were greater than in the DIP+DL and DIP+IL groups $(P=0.0006-0.03)$. However, for the g-ratio, smaller values were observed in the IIP+IL group compared with those obtained in the DIP + DL and DIP + IL groups in both the gingiva and the bone regions around the implants $(P<0.04)$. This difference could be related to an increased degree of myelination. However, no significant differences in measured fibre diameter, axon diameter or g-ratio were observed when compared with delayed implant placement, with or without immediate implant loading $(\mathrm{DIP}+\mathrm{DL} v s . \mathrm{DIP}+\mathrm{IL}, P>0.05)$.

\section{DISCUSSION}

To the best of our knowledge, this is the first time that a histomorphological change in myelinated nerve fibres following different loading protocols in the peri-implant hard and soft tissues has been described in a randomized controlled animal experiment. Moreover, this study focused on the clinical hypothesis that osseoperception of dental implants could potentially be improved by applying an immediate implant placement and loading protocol. The present findings may be of particular importance for understanding the underlying mechanisms of osseoperception and for optimising the physiological integration of implant-supported prostheses and its clinical significance.

To minimize the potential bias and variations (e.g., dynamic loading forces in the mouth) between experimental animals, a split-mouth 
design was used in this study to simplify the comparison under standardized healing and test conditions. Based on this unique design with randomized implant recipient site selection and isolated loading conditions of individual implants, the potential carryover effects could be excluded. $^{22}$

After a 10-month complete decalcification process, we were able to confirm the presence of myelinated nerve fibres adjacent to the implants and quantified their distribution features through maintaining the fine structure of the nerve fibres. As cytoskeletal elements in the axon and also as sympathetic neurotransmitters, ${ }^{23}$ NF and NPY were detected in the same nerve fibres. It has been suggested that these regenerated nerves next to the bone trabeculae could have a sensory and/or sympathetic origin, which is similar to the innervation in the tooth pulp. ${ }^{24}$ The transmission electron microscopy (TEM) observations achieved in this study were similar to those from an ultrastructure study supporting the possibility of sympathetic modulation of peri-implant mechanoreceptors. ${ }^{25}$ Notwithstanding the regularly identified mitochondria, which were assumed to exhibit A-delta nerve ending characteristics in this TEM observation, ${ }^{26}$ such TEM morphometric parameters alone are insufficient to further distinguish between A-beta and A-delta fibres. Neurophysiological aspects such as the conduction velocity of these nerve fibres would be indispensable for further differentiation. ${ }^{27}$

In the present study, myelinated nerve fibres reached the highest density in the gingival and apical regions for all groups, which was most likely due to the abundant local blood supply and innervation originally present in the PDL. Similarly, it was reported that the apical region of the natural tooth is highly innervated where the PDL received the most loading. ${ }^{2,19}$ The differences in nerve density between the left and right sides of the jaws were not significant, which was consistent with another morphological study. ${ }^{28}$ Owing to the small sample size in the maxilla, there was only a tendency towards higher nerve density around mandible implants than around maxilla implants. Nevertheless, this trend added histological evidence to the clinical observation that mandibular teeth and implants may be more sensitive to tactile tests. ${ }^{29}$

Previous morphometric studies that reported the existence of free nerve endings in the peri-implant tissues were mostly qualitative or semiquantitative, ${ }^{30}$ without any comprehensive comparison between various implant protocols. The main results found in our study were a higher nerve density and greater fibre and axon diameters for implants immediately placed and loaded. It should be mentioned that not all comparisons reached statistical significance, yet the limits related to animal research, sample size and animal variability may have prevented those comparisons from reaching significance. The fact that the fibre and axon diameters increased when immediately placed implants were followed by immediate loading may indicate better peri-implant innervation recovery. ${ }^{31-32}$ Although the mechanism is still unclear, it can be assumed that immediate implant placement after extraction leaves the nerves in place with activation that prevents degeneration. Upon implant loading, sufficient stimulation of dedifferentiated Schwann cells and/or activation of peri-implant nerve signals might occur to promote peri-implant nerve regeneration. Furthermore, these immediately placed and loaded implants also showed thicker myelin sheaths, in accordance with previous research. ${ }^{33}$

However, a higher innervation density together with a smaller fibre diameter and g-ratio was presented in the implant groups in comparison with the natural extraction healing group. It can be hypothesized that myelinated nerve fibres become more branched and smaller as they receive mechanical loading transmitted via the implant surface.
As a consequence, the fibre diameter was smaller than those observed in the natural healing sites. Moreover, the g-ratio could significantly decrease when regeneration became stabilized in a long-term study. ${ }^{21}$ Despite a shorter loading time in DIP $+\mathrm{DL}$, the regeneration of vascular and neural elements in the PDL was almost completed 4 weeks after reimplantation. ${ }^{34}$ Whether these regenerated nerves around implants could continue to mature and develop to axons with larger fibre diameters remains to be elucidated in further studies using longer follow-up periods.

However, it is worth noting some of limitations of this study. Firstly, as we were aiming to compare immediate $v s$. delayed implant loading protocols, an unloaded implant control group was not included for comparison. Nevertheless, this concern might be addressed by the fact that more free nerve endings have already been found around delayed loaded implants compared with non-loaded control implants after a 3-month observation period. ${ }^{17}$ Secondly, despite the neurophysiological and psychophysical evidence, it is not yet fully clear whether those differences in morphometric parameters resulted in the actual sensory activation with clinical significance. There is evidence that neural receptors around implants could evoke sensory nerve action potentials of the inferior alveolar nerve stem following an electrical stimulus ${ }^{35}$ or could possess a higher tactile threshold after implant rehabilitation. ${ }^{11}$ The morphological findings in this study would, in turn, promote related mechanism studies and hypotheses, such as local grafts of Schwann cells or calcitonin gene-related peptide-alpha to improve the regeneration of peripheral nerve fibres after dental implant surgery. ${ }^{36-37}$ Finally, this study provided a general animal model for the assessment of re-innervation around implants, yet precisely defined loading frequency, implant surface designs, implant-supported restorations and masticatory behaviours were not included, which precludes the generalisability of our findings.

In conclusion, based on the histomorphometric exploration of the origin and distribution of peri-implant innervation, this study helped uncover the tendency that the immediately placed and loaded implant group may have an improved peri-implant innervation pattern, including higher nerve density, larger fibre diameter and axon diameter, and more axonal myelination. The current histological findings on these regenerated nerve fibres may expand the concept of osseoperception to more challenging levels and may evolve with osseointegration in the oral environment and in ear-nose-throat and orthopaedics specialties. Further research examining the functional role of the identified nerve fibres and establishing the potential morpho-physiological relationship is clearly required.

\section{ACKNOWLEDGEMENTS}

We thank Jeanine Santermans and Marc Jans for their technical assistance. This study was supported by the Natural Science Foundation of China (81000459) and the Chinese Scholarship Council.

1 Jacobs R, van Steenberghe D. Role of periodontal ligament receptors in the tactile function of teeth: a review. J Periodontal Res 1994; 29(3): 153-167.

2 Trulsson M. Sensory-motor function of human periodontal mechanoreceptors. J Oral Rehabil 2006; 33(4): 262-273.

3 Svensson KG, Grigoriadis J, Trulsson M. Alterations in intraoral manipulation and splitting of food by subjects with tooth- or implant-supported fixed prostheses. Clin Oral Implants Res 2013; 24(5): 549-555.

4 Mason AG, Holland GR. The reinnervation of healing extraction sockets in the ferret. J Dent Res 1993; 72(8): 1215-1221.

5 Brånemark PI. Osseointegration: biotechnological perspetive and clinical modality// Brånemark $\mathrm{PI}$, Rydevik $\mathrm{B}$, Skalak RS eds. Proceedings of the osseointegration in skeletal reconstruction and joint replacement. Carol Stream: Quintessence Publishing, 1997. 
6 van Steenberghe D. From osseointegration to osseoperception. J Dent Res 2000; 79(11): 1833-1837.

7 Jacobs R, Van Steenberghe D. From osseoperception to implant-mediated sensorymotor interactions and related clinical implications. J Oral Rehabil 2006; 33(4): 282-292.

8 Jacobs R. Neurological versus psychophysical assessment of osseoperception//Jacobs R ed. Proceedings of the osseoperception. Leuven: Catholic University Leuven, 1998 75-88.

9 Jacobs R, van Steenberghe D. Comparative evaluation of the oral tactile function by means of teeth or implant-supported prostheses. Clin Oral Implants Res 1991; 2(2): 75-80.

10 Enkling N, Heussner S, Nicolay C et al. Tactile sensibility of single-tooth implants and natural teeth under local anesthesia of the natural antagonistic teeth. Clin Implant Dent Relat Res 2012; 14(2): 273-280.

11 Kazemi M, Geramipanah F, Negahdari R et al. Active tactile sensibility of single-tooth implants versus natural dentition: a split-mouth double-blind randomized clinica trial. Clin Implant Dent Relat Res 2013; doi: 10.1111/cid.12053. [Epub ahead of print].

12 Habre-Hallage P, Dricot L, Jacobs R et al. Brain plasticity and cortical correlates of osseoperception revealed by punctate mechanical stimulation of osseointegrated oral implants during fMRI. Eur J Oral Implantol 2012; 5(2): 175-190.

13 Habre-Hallage $P$, Dricot $L$, Hermoye $L$ et al. Cortical activation resulting from the stimulation of periodontal mechanoreceptors measured by functional magnetic resonance imaging (fMRI). Clin Oral Investig 2014; doi: 10.1007/s00784-0131174-1. [Epub ahead of print].

14 Lambrichts I. Histological and ultrastructural aspects of bone innervation//Jacobs R ed. Proceedings of the osseoperception. Leuven: Catholic University Leuven, 1998: 13-20.

15 Suzuki Y, Matsuzaka K, Ishizaki K et al. Characterization of the peri-implant epithelium in hamster palatine mucosa: behavior of Merkel cells and nerve endings. Biomed Res 2005; 26(6): 257-269.

16 Yamaza T, Kido MA, Wang B et al. Distribution of substance $P$ and neurokinin-1 receptors in the peri-implant epithelium around titanium dental implants in rats. Cell Tissue Res 2009; 335(2): 407-415.

17 Wada S, Kojo T, Wang YH et al. Effect of loading on the development of nerve fibers around oral implants in the dog mandible. Clin Oral Implants Res 2001; 12(3): 219224.

18 Huang Y, van Dessel J, Liang X et al. Effects of immediate and delayed loading on periimplant trabecular structures: a cone beam CT evaluation. Clin Implant Dent Relat Res 2013; doi: 10.1111/cid.12063. [Epub ahead of print].

19 Huang Y, Corpas LS, Martens W et al. Histomorphological study of myelinated nerve fibres in the periodontal ligament of human canine. Acta Odontol Scand 2011; 69(5): 279-286.

20 Weiner S, Klein M, Doyle JL et al. Identification of axons in the peri-implant region by immunohistochemistry. Int J Oral Maxillofac Implants 1995; 10(6): 689-695.

21 Muratori L, Ronchi G, Raimondo S et al. Can regenerated nerve fibers return to normal size? A long-term post-traumatic study of the rat median nerve crush injury model. Microsurgery 2012; 32(5): 383-387.

22 Lesaffre E, Philstrom B, Needleman I et al. The design and analysis of split-mouth studies: what statisticians and clinicians should know. Stat Med 2009; 28(28): 3470-3482.
23 Georgiou M, Bunting SC, Davies HA et al. Engineered neural tissue for peripheral nerve repair. Biomaterials 2013; 34(30): 7335-7343.

24 Sacerdote $P$, Levrini L. Peripheral mechanisms of dental pain: the role of substance $P$. Mediators Inflamm 2012; 2012: 951920.

25 Lambrichts I, Creemers J, vandenabeele F et al. Possible sympathetic modulation of periodontal ligament mechanoreceptors. An ultrastructural study. Biol Cell 1995; 84(3): 230-231.

26 Vandenabeele F, Creemers J, Lambrichts I et al. Encapsulated Ruffini-like endings in human lumbar facet joints. J Anat 1997; 191(Pt 4): 571-583.

27 Cadden SW, Lisney SJ, Matthews B. Thresholds to electrical stimulation of nerves in cat canine tooth-pulp with A beta-, A delta- and C-fibre conduction velocities. Brain Res 1983; 261(1): 31-41.

28 Long A, Loescher AR, Robinson PP. A quantitative study on the myelinated fiber innervation of the periodontal ligament of cat canine teeth. J Dent Res 1995; 74(6): 1310-1317.

29 Yoshida K. Tactile threshold for static and dynamic loads in tissue surrounding osseointegrated implants//Jacobs R ed. Proceedings of the osseoperception. Leuven: Catholic University Leuven, 1998: 143-156.

30 Huang $\mathrm{Y}$, Jacobs $\mathrm{R}$, van Dessel $\mathrm{J}$ et al. A systematic review on the innervation of periimplant tissues with special emphasis on the influence of implant placement and loading protocols. Clin Oral Implants Res 2014; doi: 10.1111/clr.12344. [Epub ahead of print].

31 de Medinaceli L. Interpreting nerve morphometry data after experimental traumatic lesions. J Neurosci Methods 1995; 58(1/2): 29-37.

32 Wolthers M, Moldovan M, Binderup T et al. Comparative electrophysiological, functional, and histological studies of nerve lesions in rats. Microsurgery 2005; 25(6): 508-519.

33 Ikeda M, Oka Y. The relationship between nerve conduction velocity and fiber morphology during peripheral nerve regeneration. Brain Behav2012; 2(4): 382-390.

34 Yamada $\mathrm{H}$, Maeda $\mathrm{T}$, Hanada $\mathrm{K}$ et al. Re-innervation in the canine periodontal ligament of replanted teeth using an antibody to protein gene product 9.5: an immunohistochemical study. Endod Dent Traumatol 1999; 15(5): 221-234.

35 Qiao SC, Lv XF, Zhuang LF et al. [Animal study of sensory function of nerve fibers surrounding dental implant.] Shanghai Kou Qiang Yi Xue 2011; 20(2): 119-124. Chinese.

$36 \mathrm{Ma} \mathrm{L}$, Xiang L, Yao Y et al. CGRP-alpha application: a potential treatment to improve osseoperception of endosseous dental implants. Med Hypotheses 2013; 81(2): 297 299.

37 Yuan Q, Gong P, Tan Z. Schwann cell graft: a method to promote sensory responses of osseointegrated implants. Med Hypotheses 2007; 69(4): 800-803.

\section{(c) $($ ) $€$}

This work is licensed under a Creative Commons Attribution-

NonCommercial-NoDerivs 3.0 Unported License. The images or other third party material in this article are included in the article's Creative Commons license, unless indicated otherwise in the credit line; if the material is not included under the Creative Commons license, users will need to obtain permission from the license holder to reproduce the material. To view a copy of this license, visit http://creativecommons.org/licenses/ by-nc-nd/3.0/

Supplementary information for this article can be found on International Journal of Oral Science's website (http://www.nature.com/ijos). 